COMMENT. ADEM is a post- or para-infectious illness that is usually preceded by a febrile upper respiratory viral infection (URI) or vaccination, and follows a monophasic course with recovery in $90 \%$. ADEM is distinguished from MS by long-term clinical followup, absence of relapsing course and new MRI lesions, and absent oligoclonal bands in the CSF (see Ped Neur Briefs Nov 2002;16:81-82, for commentary on long-term study of 84 children with $\mathrm{ADEM}$ ). Coronavirus ( $\mathrm{HCoV})$, a common cause of URI, has not previously been associated with ADEM, although a chronic demyelinating disorder resembling MS occurs with $\mathrm{HCoV}$ infection in the mouse model. HCoV RNA has been demonstrated in the CSF and brain of MS patients, but also in autopsy specimens of controls without neurologic disease. The role of coronavirus in demyelinating disease is undetermined.

\title{
VARICELLA ZOSTER VIRUS OPTIC NEURITIS
}

A 13-year-old male presented with a corticosteroid-sensitive, varicella zoster virus (VZV)-related rapid loss of vision and bilateral disc edema after a febrile illness, and the case is reported from the University of Pittsburgh School of Medicine, Pittsburgh, PA. Increasingly slow and prolonged corticosteroids taper was required to avoid relapse of visual function over a 1 year period. VZV seroconversion occurred late in the course of the disease. Neuroimaging was unrevealing. CSF was under increased pressure $(260 \mathrm{~mm} \mathrm{H} 2 \mathrm{O})$, protein $60 \mathrm{mg} / \mathrm{dL}$, glucose $48 \mathrm{mg} / \mathrm{dL}$, WBC $108 \mathrm{~mm} 3$ ( $84 \%$ lymphocytes), absent oligoclonal bands, IgG index $0.65(\mathrm{~N}<0.7)$, and polymerase chain reaction for other viruses was negative. Initial treatment with acetazolamide was unsuccessful. When prednisone was substituted, disc edema slowly improved and visual acuity became normal. Tapering of prednisone after 6 weeks was followed by recurrence of disc edema and visual loss, MRI showed T2-signal hyperintensities in the right insular and frontal cortex regions, and in the upper cervical cord. The serum and CSF VZV complement fixation assays were now positive. Treatment with intermittent steroids and oral acyclovir was followed by remission and relapse over an 18month period. At 1 year, the dose of prednisone was $20 \mathrm{mg}$ every other day, visual acuity was $20 / 20$, color perception $80 \%$, but optic discs were pale and pupil responses were defective. Otherwise, the neurologic exam was normal. (Pless ML, Malik SI. Relapsingremitting, corticosteroid-sensitive, varicella zoster virus optic neuritis. Pediatr Neurol Nov 2003;29:422-424). (Respond: Misha L Pless MD, Director, Neuro-Ophthalmology; Eye and Ear Institute, 203 Lothrop St, Pittsburgh, PA 15213).

COMMENT. VZV may cause neurologic disease without a rash (zoster sine herpete), and complications associated with viral reactivation include optic neuritis, aseptic neuritis, myelitis, and encephalitis. Immunological mechanisms are considered likely. Since seroconversion to VZV occurred within 2 months of onset in the above case, VZV was considered the most likely cause of the optic neuritis, and multiple sclerosis or ADEM was a less plausible explanation.

VZV is a human herpes virus that causes varicella as a primary infection after which the virus lies dormant in the trigeminal and dorsal root ganglia. With reactivation, the virus causes herpes zoster, sometimes followed by post-herpetic neuralgia. In a review of advances in neurological infectious diseases, Kennedy PGE (Lancet Neurology Jan 2004;3:13) cites a case of zoster sine herpete in a woman with a 13 month history of right maxillary trigeminal 
nerve pain, without rash, with a mass in the trigeminal ganglion and chronic ganglionitis (Hevner R et al. Lancet Neurol 2003;2:567-571).

Ocular manifestations of the congenital varicella syndrome were described in 3 children seen at Great Ormond Street Hospital, London, UK (Lambert SR et al. Arch Ophthalmol 1989;107:52-56) and reviewed in Progress in Pediatric Neurology I, PNB Publ, 1991;424-5). Ocular abnormalities followed a maternal varicella infection in the second trimester of pregnancy, and included chorioretinitis, atrophy of optic discs, cataract, and Horner's syndrome. Neurologic complications included bulbar palsy, hemiparesis, learning disorder, and psychomotor retardation.

Varicella with delayed hemiplegia is described in 4 children reported from Japan. (Ichiyama T et al. Pediatr Neurol 1990;6:279-281; and PPN I, PNB 1991;425). Cerebral angiitis with occlusion of the middle cerebral artery was cited as the cause. Neurologic complications of varicella are due to viremia with encephalitis, post-exanthematous encephalitis or cerebral angiitis. Cerebellar ataxia is the most frequent neurologic complication of varicella and hemiplegia is unusual.

\section{IMMUNOTHERAPIES IN RASMUSSEN'S ENCEPHALITIS}

Corticosteroids, IV immunoglobulins (IVIg), cyclophosphamide, therapeutic plasma exchange (TPE), and protein A IgG immunoadsorption (PAI) were evaluated in 15 patients with confirmed Rasmussen's encephalitis (RE) and reported from various centers in Italy. Steroids were used for status epilepticus or worsening seizures. In 6/11 patients steroid treatment temporarily reduced seizure frequency. All attempts to withdraw prednisone resulted in seizure exacerbation, and treatment was maintained until surgery. IVIg in 11 patients was partially effective in 6. Cyclophosphamide was ineffective in 4 patients treated, 1 developing severe leukopenia. TPE was effective in the control of status epilepticus in 1 of 5 patients treated. $P A I$ given chronically initially controlled status epilepticus and improved the neurologic condition in 3 patients treated, but the effect was not sustained. Surgery (hemispherotomy or hemispherectomy) in 13 patients at $5.2+/-3.9$ years after disease onset controlled seizures initially, and antiepileptic drugs were withdrawn in 4 and reduced in 7 . After surgery, the neurologic condition improved in all patients. Surgery is the only treatment that halts disease progression. Immunomodulatory therapy is considered when surgery is not feasible, in late-onset patients with slow progression, and in the infrequent case of bilateral disease. (Granata T, Fusco L, Gobbi G et al. Experience with immunomodulatory treatments in Rasmussen's encephalitis. Neurology December (2 of 2) 2003;61:1807-1810). (Reprints: Dr Tiziana Granata, Division of Child Neurology, Instituto Nazionale Neurologico C. Besta, Via Celoria 11, 20133 Milan, Italy).

COMMENT. The authors propose immunotherapy to delay surgery in patients with 1) late onset (adolescent or adult) RE with slower and milder course than the typical childhood-onset form; 2) dominant hemisphere involvement and slow progression, when surgery is contraindicated; 3) suspected cases of RE without deterioration and atrophy; 4) bilateral RE, and 5) severe cases requiring stabilization before surgery. Surgery is the preferred and optimal treatment to control seizures and halt neurologic deterioration. Bolus corticosteroids and PAI block status epilepticus. IVIg may have a role in adult patients but is ineffective in childhood. 